Check for updates

Cite this: RSC Adv., 2018, 8, 6978

Received 1st November 2017

Accepted 5th February 2018

DOI: $10.1039 / \mathrm{c} 7 \mathrm{ra12008 \textrm {g }}$

rsc.li/rsc-advances

\section{Factors affecting the structure of lyotropic liquid crystals and the correlation between structure and drug diffusion}

\begin{abstract}
Yiming Huang (D) and Shuangying Gui*abc
Lyotropic liquid crystals (LLCs) formed by the self-assembly of amphiphilic molecules in a solvent (usually water) have attracted increasingly greater attention in the last few decades, especially the lamellar phase $\left(L_{\alpha}\right)$, the reversed bicontinuous cubic phase $\left(Q_{2}\right)$ and the reversed hexagonal phase $\left(H_{2}\right)$. Such phases offer promising prospects for encapsulation of a wide range of target molecules with various sizes and polarities owing to the unique internal structures. Also, different structures of mesophases can give rise to different diffusion coefficients. The bicontinuous cubic phase and the hexagonal phase have been demonstrated to control and sustain the release of active molecules. Furthermore, the structures are susceptible to many factors such as water content, temperature, $\mathrm{pH}$, the presence of additives etc. Many researchers have been studying these influencing factors in order to accurately fabricate the desired phase. In this paper, we give a review of the characteristics of different structures of liquid crystalline phases, the influencing factors on the phase transition of liquid crystals and the relationship between structures of LLC and drug diffusion. We hope our review will provide some insights into how to manipulate in a controlled manner the rate of incorporating and transferring molecules by altering the structure of lyotropic mesophases.
\end{abstract}

\section{Introduction}

Sustained-release preparations are able to maintain drug concentrations in the range of therapeutic concentrations for a prolonged time frame. ${ }^{\mathbf{1} 2}$ This can reduce the frequency of administration and toxicity and minimize side effects compared with general preparations.

LLCs, based on the self-assembly of amphiphilic molecules in excess water, have received considerable attention as a carrier for sustained release drugs. ${ }^{3-5}$ The internal structures of the LLCs are composed of a hydrophilic domain, lipophilic domain and lipid layer. Therefore, LLCs can encapsulate a wide range of active drugs (i.e. hydrophilic, hydrophobic, and amphiphilic) and protect them from hydrolysis and enzymolysis. ${ }^{6-9}$ Normally, hydrophilic drugs are located near the lipid polar head or in the water channels, while the lipophilic drugs are loaded in the lipid layer and the amphiphilic drugs are at the lipid layer interface. ${ }^{\mathbf{1 0}}$

The most commonly studied types of LLCs are the lamellar, the bicontinuous cubic, the hexagonal mesophase and the

${ }^{a}$ Department of Pharmacy, Anhui University of Chinese Medicine, No. 1, Qianjiang Road, Hefei, Anhui 230012, China. E-mail: guishy0520@126.com; Fax: +86-55168129121; Tel: +86-551-68129121

${ }^{b}$ Institute of Pharmaceutics, Anhui Academy of Chinese Medicine, Hefei, Anhui 230012, China

${ }^{c}$ Key Laboratory of Xin'an Medicine, Ministry of Education, Hefei, Anhui 230012, China micellar cubic phases. ${ }^{\mathbf{1 1 , 1 2}}$ Moreover, the release rates of loaded drugs are distinguishable in different liquid crystal phases. ${ }^{\mathbf{1 3 , 1 4}}$ It is the dimensions of the lyotropic liquid crystal structures that determine the release rate of the entrapped drug. The lamellar phase is a one-dimensional structure made up of lipid bilayers and layers of water. ${ }^{15}$ The bicontinuous cubic is a "honeycomb" structure in which the amphiphilic molecules are stacked in three-dimensional space. ${ }^{16}$ The two water channels are consecutive but not crossed and are separated by a lipid bilayer. Therefore, the cubic phase can sustain the release of active molecules. ${ }^{17}$ In addition, the hexagonal mesophase is arranged in hexagons by a closed cylindrical micelle. ${ }^{18}$ The release rate of drugs is slower than in the bicontinuous cubic phase. ${ }^{19,20}$ As for the reversed micellar cubic of $F d 3 m$, it is a closed structure consisting of closely packed inverse micelles of two sizes. ${ }^{21} \mathrm{Fd} 3 \mathrm{~m}$ has been of increasing interest to researchers because the release of hydrophilic substances from the reversed micellar cubic phase is slower than that from the reversed hexagonal phase. ${ }^{22}$

The structures of the LLCs are affected by many factors including temperature, ${ }^{19} \mathrm{pH},{ }^{23-26}$ light, ${ }^{27-32}$ magnetic field, ${ }^{33,34}$ the type of amphiphilic, ${ }^{35}$ water content ${ }^{36,37}$ and additives. ${ }^{38,39}$ Therefore, by altering the preparation conditions, desired phase structures can be achieved and intelligent carriers can be fabricated. Fong et al. ${ }^{19}$ have shown the possibility of using temperature to trigger release. More specifically, they successfully switched the hexagonal mesophase to the bicontinuous cubic phase by changing the temperature above or below 
physiological temperature. The in vitro release studies showed that the release rate of hydrophilic drug glucose from the hexagonal phase was slower than that from the cubic phase. There are also many studies using $\mathrm{pH}$ to regulate the release rate of drug according to the $\mathrm{pH}$ changing within human body. ${ }^{\mathbf{4 0 1}}$ For example, linoleic acid is susceptible to $\mathrm{PH}$, so addition of linoleic acid to $\mathrm{MLO} /$ water or $\mathrm{MO} /$ water can control the formation of mesophases by controlling the $\mathrm{pH}$, thereby affecting drug release. ${ }^{23,24}$

Besides, some researchers have also tried to change the internal structures of LLCs by encapsulating reconstitute membrane proteins within to control the release of the drug. ${ }^{26,42,43}$ Zabara et al. ${ }^{\mathbf{2 6}}$ designed a novel perforated bicontinuous cubic phase $(\mathrm{Pn} 3 \mathrm{~m})$, which was formed by adding outer membrane protein $\mathrm{F}$ (OmpF) to monolinolein (MLO)-water system. The results showed that the presence of OmpF did not alter the structure of $P n 3 m$ compared with the Pn $3 m$ formed by MLO-water. The OmpF proteins are located at the lipid bilayer of $P n 3 m$ cubic phase and therefore they can connect the two parallel sets of aqueous channels. Structural data showed that the OmpF contains a constriction site with two amino acid halfrings (one positively charged and the other negatively charged) which lead to sensitivity to $\mathrm{PH}$. Therefore, OmpF acted as "pH-triggered release switch". At $\mathrm{pH}=7.4$, the model drug glucose in OmpF-doped mesophase was released faster than in the blank (without OmpF). However, when the $\mathrm{pH}=4.8$, the release rates of glucose in two kinds of $P n 3 m$ were similar.

In this review, we initially briefly introduce the different structures of LLCs. We then offer an overview of the influencing factors on the phase structures, including prescription factors (i.e. amphiphilic molecules, water content, additives) and external factors (i.e. temperature, pressure, light, magnetic field). In the end, the relationship between structure and drug diffusion is discussed.

\section{The structure characteristic of lyotropic liquid crystals}

Generally, with the an increase amphiphilic concentrations, different well-defined structures are derived, such as the normal micelle (oil in water), the normal cubic phase, the normal hexagonal phase, the lamellar phase, the reversed cubic phase (water in oil) and the reversed hexagonal phase. Additionally, the reversed cubic phases can be divided into two categories, the reversed bicontinuous cubic phase and the reversed micellar cubic phase. Besides, a nomenclature for mesophases was proposed $\mathbf{4}^{\mathbf{4 4 5}}$ using various capital letter and subscripts. For example, L stands for the lamellar phase, Q for the cubic phase, $\mathrm{V}$ for the bicontinuous cubic phase, I for the micellar cubic phase and $\mathrm{H}$ for the hexagonal phase. Furthermore, subscripts I (1) and II (2) represent for normal and reversed phases respectively. Among all of the structures, the lamellar phase, the reversed cubic phase and the reversed hexagonal phase have received much attention for their highly ordered internal structures, which gives these phases great the potential as drug delivery systems. These common structures of LLCs diagram are presented in Fig. 1.

\subsection{The lamellar phase}

The lamellar phase extensively exists in organisms. ${ }^{46}$ For example, it is the basic building block of cell membranes. The lamellar phase is a planar structure, consisting of lipid bilayers separated by water, where the polar head groups of the

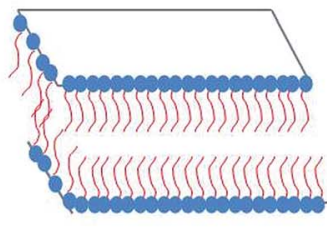

(a)

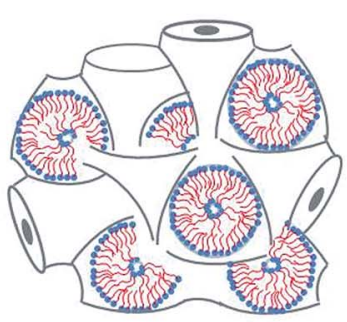

(d)

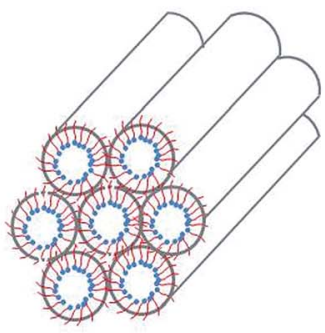

(b)

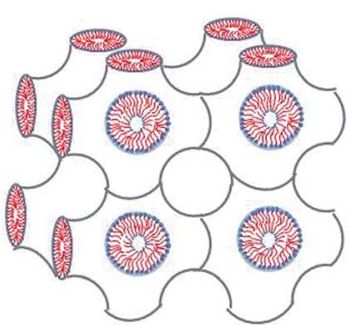

(e)

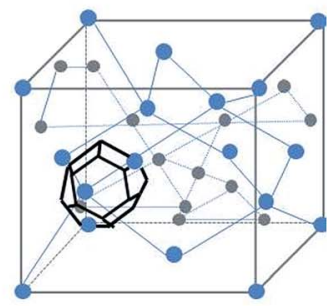

(c)

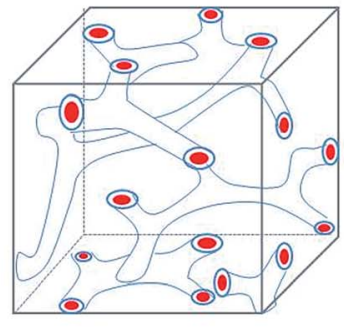

(f)

Fig. 1 Schematic representation of the lyotropic liquid crystalline phases commonly found in neutral lipid/water systems. (a) Lamellar phase (b) reverse hexagonal phase (c) reversed micellar cubic of $F d 3 m$ (d) reversed bicontinuous cubic ( $/ m 3 m$ ) (e) reversed bicontinuous cubic (Pn3m) (f) reversed bicontinuous cubic (la3d). 
Table 1 Physical properties of common lyotropic liquid crystals

\begin{tabular}{llll}
\hline Types of LLC phase & Optical properties & CPLM & SAXS \\
\hline Lamellar phase & Anisotropic & Streaky-like & $1: 2: 3: 4$ \\
Im3m & Isotropic & Dark field & $\sqrt{2}: \sqrt{4}: \sqrt{6}: \sqrt{8}$ \\
Pn3m & Isotropic & Dark field & $\sqrt{2}: \sqrt{3}: \sqrt{4}: \sqrt{6}: \sqrt{8}: \sqrt{9}$ \\
Ia3d & Isotropic & Dark field & $\sqrt{6}: \sqrt{8}: \sqrt{14}: \sqrt{16}: \sqrt{20}: \sqrt{22}$ \\
Fd3m & Isotropic & Dark field & $\sqrt{3}: \sqrt{8}: \sqrt{11}: \sqrt{16}: \sqrt{19}$ \\
Reverse hexagonal & Anisotropic & Fan-like & $1: \sqrt{3}: 2: \sqrt{7}$ \\
& & & \\
\hline
\end{tabular}

amphiphilic molecules associate and are in contact with water directly, while the hydrophobic tails are away from water. ${ }^{\mathbf{4 7 , 4 8}}$ The rheology gives the information that the lamellar structure is less viscous compared with the hexagonal structure. Furthermore, the lamellar phase can be characterized by Crossed Polarized Light Microscopy (CPLM), exhibiting "streaky" or "mosaic" like texture. In addition, Bragg peaks with relative positions at the ratios of $1: 2: 3: 4$ are obtained from the Small-Angle X-ray Scattering (SAXS) measurement.

\subsection{The reversed bicontinuous cubic}

The reversed bicontinuous cubic phase is complex and comprises two continuous but nonintersecting water channels separated by a curved bicontinuous lipid bilayer. ${ }^{49}$ Based on the theory of infinite periodic minimal surface (IPMS), the reversed bicontinuous cubic phase can be regarded as a three dimensional periodic lipidic bilayer with zero mean curvature at each point. It should be noted that the reversed bicontinuous cubic phase can be divided into three types based on X-ray crystallographic studies, namely, the primitive lattice $\left(\operatorname{Im} 3 m, \mathrm{Q}_{229}\right)$, the double-diamond lattice $\left(P n 3 m, \mathrm{Q}_{224}\right)$, and the gyroid lattice (Ia3d, $\mathrm{Q}_{230}$ ). Furthermore, the bulk phase is a transparent, viscous gel, which can be characterized by CPLM and SAXS. ${ }^{\mathbf{5 0 , 5 1}}$ Under the CPLM, all types of the bulk phase do not display any optical textures. However, the SAXS can be used to distinguish different types. The ratios of Bragg peaks at different positions are as follow: $\operatorname{Im} 3 m(\sqrt{2}: \sqrt{4}: \sqrt{6}: \sqrt{8})$, Pn3m ( $\sqrt{2}: \sqrt{3}: \sqrt{4}: \sqrt{6}: \sqrt{8}: \sqrt{9}), \quad$ Ia3d $(\sqrt{6}: \sqrt{8}: \sqrt{14}: \sqrt{16}: \sqrt{20}: \sqrt{22})$.

\subsection{The reverse hexagonal phase}

The reverse hexagonal phase is made up by a large quantity of densely packed water filled cylindrical micelles, following a long-range order two-dimensional lattice. ${ }^{52}$ Significantly, the diameter of the cylindrical micelle is about 1-2 nm, containing $30-60 \%$ water by weight. Furthermore, it is reported that the water inside the cylindrical micelles is not in contact with the water outside directly. The reverse hexagonal phase is less viscous than the reversed bicontinuous cubic. In other words, the reverse hexagonal phase is of intermediate viscosity compared with the lamellar phase and the reversed bicontinuous cubic phase. By CPLM, the reverse hexagonal phase appears "fan-like" textures and the ratios of Bragg peaks at different positions are $1: \sqrt{3}: 2: \sqrt{7}$ according to the SAXS measurement..$^{53,54}$

\subsection{The reversed micellar cubic}

The reversed micellar cubic is also referred as the discontinuous cubic phase which consists of micelles arranged in a cubic lattice. There are two main micellar cubic phases: $F d 3 m$ and $F m 3 m$. The $F d 3 m$ micellar cubic phase is a closed structure composed of micelles of two different sizes organized in a double diamond network. ${ }^{21}$ The Bragg peaks are in the ratios of $\sqrt{3}: \sqrt{8}: \sqrt{11}: \sqrt{16}: \sqrt{19} .^{55,56}$ The $F d 3 m$ phase is currently the most extensively studied structure for sustained drug release. The physical properties of $F d 3 m$ and the mesophases mentioned above are summarized in Table 1.

Reverse micellar cubic mesophase of symmetry Fm3m was reported by I. Martiel which consists of ternary mixtures of soy bean phosphatidylcholine (PC), water, and an organic solvent, including cyclohexane, $(R)-(+)$-limonene, and isooctane. ${ }^{57}$ It is composed of a compact packing of remarkably large and monodisperse reverse micelles in a face-centered cubic (fcc) lattice. Therefore, the drug loading may increase. In addition, Fm3m is stable in equilibrium with excess water, so in principle it can be dispersed into particles for use as a delivery system.

\section{The influencing factors of phase transition}

Studies have shown that phases can be altered by many factors. Therefore, in the recent years, scientific efforts have converged toward studying the influencing factors in order to control the preparation conditions of the desired phases. The influencing factors can be divided into external factors, such as temperature, pressure, light, and magnetic field and prescription factors, such as presence of amphiphilic molecules, water content and the third additives. The mechanism of the structural transitions affected by factors mentioned above can be explained by the critical packing parameter (CPP), which is based on the spatial stacking of amphiphilic molecules to predict the structure of the system may be formed. ${ }^{58,59} \mathrm{CPP}$ is defined as CPP $=V / a l$, where $V$ represents the hydrophobic chain volume, $a$ represents the cross-sectional area of the hydrophilic head group, and $l$ represents the hydrophobic chain length in the molten state. According to the theory of CPP, different phases correspond to different CPP values. When CPP 
$<1$, normal phases are formed which means that the crosssectional area of the hydrophilic head group is larger than that of the hydrophobic tails, such as the normal micelle $\left(\mathrm{L}_{1}\right)$, the normal discontinuous cubic $\left(\mathrm{I}_{\mathrm{I}}\right)$ phase, the normal hexagonal $\left(\mathrm{H}_{\mathrm{I}}\right)$ phase and the normal bicontinuous cubic $\left(\mathrm{Q}_{\mathrm{I}}\right)$ phase. Conversely, reversed phases such as the reversed bicontinuous cubic $\left(\mathrm{Q}_{\mathrm{II}}\right)$ phase, the reversed hexagonal $\left(\mathrm{H}_{\mathrm{II}}\right)$ phase, the reversed discontinuous cubic $\left(\mathrm{I}_{\mathrm{II}}\right)$ phase and the reversed micelle $\left(\mathrm{L}_{2}\right)$ are developed with CPP $>1{ }^{60}$ When the polar head group area and the tail are almost equal, the lamellar phase is formed (Fig. 2).

\subsection{Prescription factors}

3.1.1 Amphiphilic molecules. Numerous amphiphilic molecules gain the ability to form lyotropic liquid crystals in water via self-assembly. Examples include phytantriol (PT), ${ }^{37,61}$ glyceryl monooleate (monoolein, GMO or MO), ${ }^{62,63}$ glyceryl monolinoleate (monolinolein, GML or MLO), ${ }^{64,65}$ oleyl glyceryl $(\mathrm{OG})^{66}$ and phytanyl glycerate (PG), ${ }^{67}$ and other lipids such as phospholipids, ${ }^{\mathbf{6 8 , 6 9}}$ phosphatidylethanolamine, ${ }^{\mathbf{7 0 - 7 2}}$ glycolipides, ${ }^{73}$ and alkyl glycerates. ${ }^{74}$ Nevertheless, the phase behavior of different amphiphilic molecules is not the same, which can be ascribed to the difference in the hydrophilic hydration intensity, the number of alkyl chains, length and the degree of unsaturation Ben J. Boyd et al. ${ }^{35}$ suggested the fact that OG and PG were able to form the reversed hexagonal phase in excess water at the physiological temperature. GMO, one of the monoglycerides and with the same molecular weight as OG, could only form the cubic phase under the same conditions.
Among the lipid materials mentioned above, GMO, MLO and PT have become the most widely used materials for encapsulation of a wide range of target molecules with various sizes and polarities.

3.1.2 Water content. Water content is an important factor affecting the formation and structure of liquid crystals. Generally, with the decrease in water content, different well-defined structures are derived, such as the normal micelle (oil in water), the normal cubic phase, the normal hexagonal phase, the lamellar phase, the reversed cubic phase (water in oil) and the reversed hexagonal phase. The phase behaviors of GMOwater, PT-water and MLO-water are depicted in Fig. 3.

Lyotropic liquid crystals are formed by adding the amphiphilic molecules to a polar solvent. Amphiphilic molecules contain a polar hydrophilic head and a non-polar hydrophobic tail. When getting in contact with water, the polar head gets close to water, while the hydrophobic tails keep away from water and are packed tightly for minimizing the free energy of the system. When the water content of the system is higher than the concentration of amphiphilic molecules, the amphiphilic molecules are tiled at the water interface. When the concentration of amphiphilic molecules exceeds the critical micelle concentration (CMC), micelles of different shapes will be formed. If the concentration continues to increase, the micelles will be further assembled into LLC with different structures. ${ }^{75,76}$

3.1.3 Additives. As shown in the discussion above, the structures of the LLCs are subject to many factors. Among them, the effect of additives or pharmaceutical compounds on phase behavior has attracted the attention of many scientists. It is worth noting that the nature of the additives has a significant

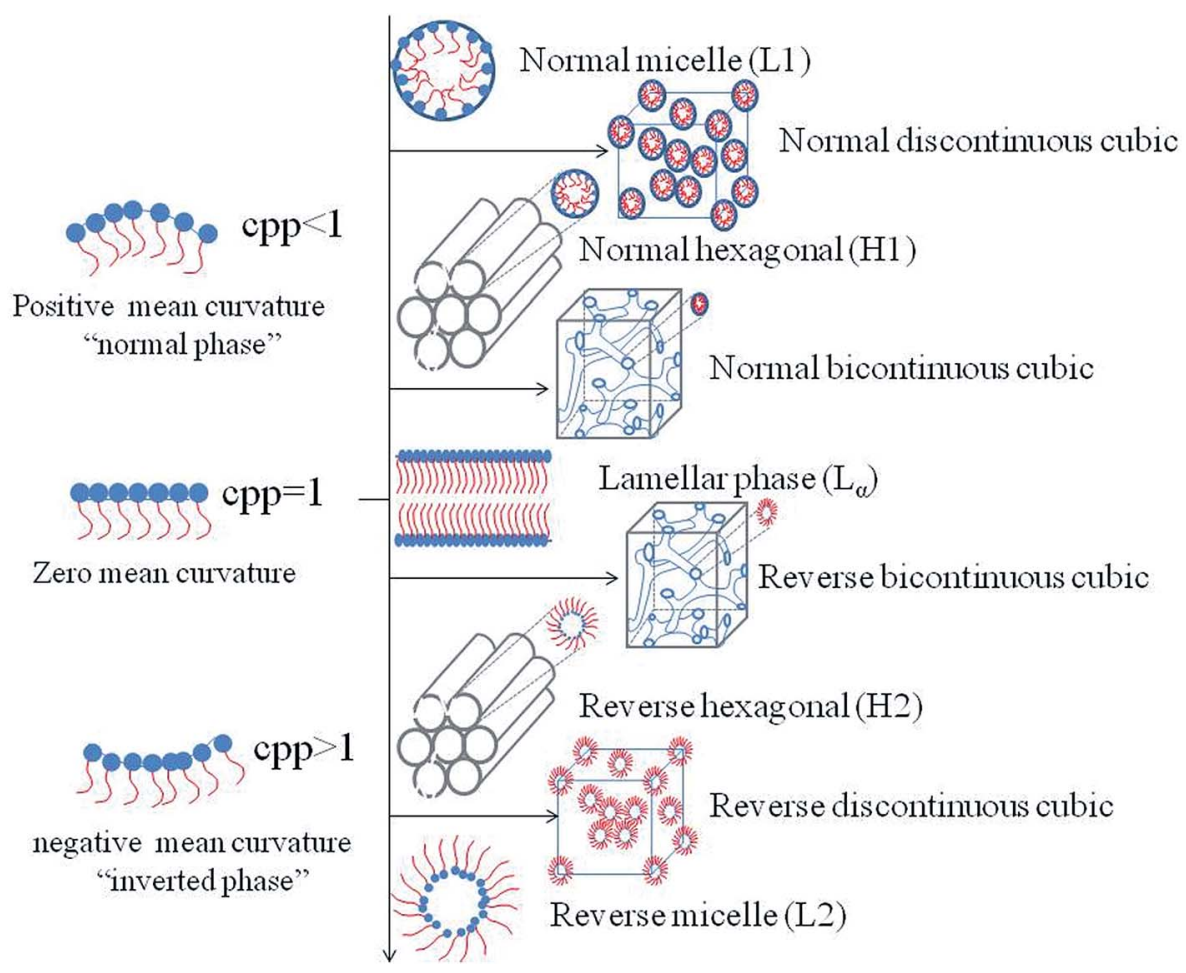

Fig. 2 Schematic representations of common structures and their corresponding CPP. 


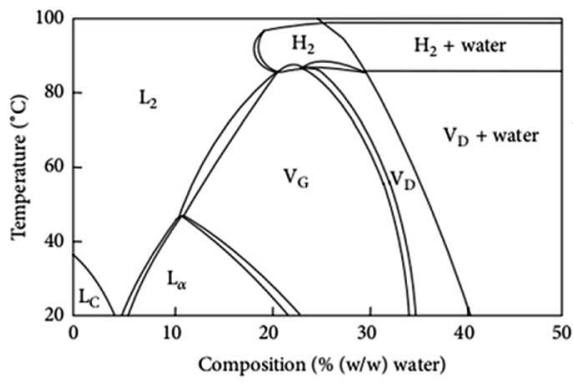

(a)

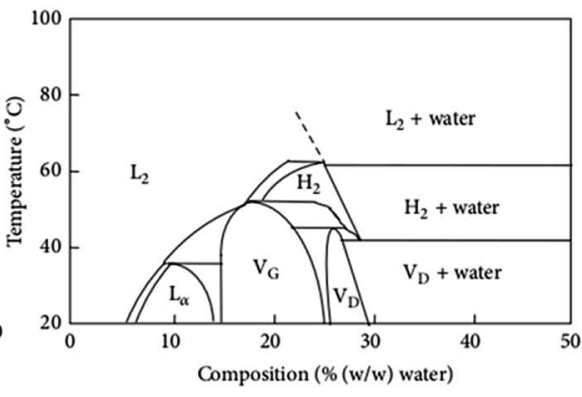

(b)

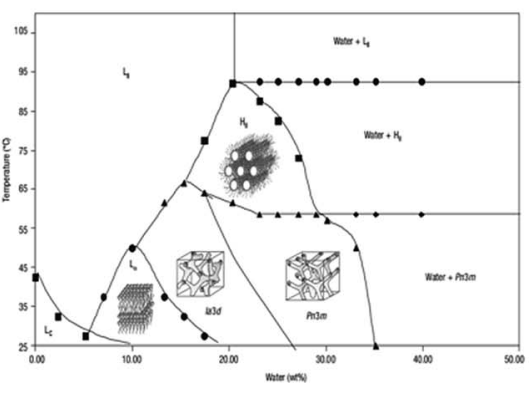

(c)

Fig. 3 The phase behaviors of GMO-water (a), ${ }^{62} \mathrm{PT}$-water (b) ${ }^{37}$ and MLO-water (c). ${ }^{77}$

impact on the phase behavior. The presence of hydrophobic compounds induces the transformation to hexagonal phase or the reversed micelle, whereas hydrophilic compounds cause the formation of the lamellar phase.

Shah and Paradkar $^{38}$ explored the effect of different hydrophilic-lipophilic balance (HLB) on the system of GMO and water. Results indicated that additives with the HLB values of $1.5,3,4$, and 5 formed the reversed hexagonal phase, while the lamellar phase was present with additives of HLB 7, 10, and 11 after 12 hours. The authors offered an explanation for these results: the lipophilic additives interacted with the tail of GMO, which in turn caused an increase in the CPP value and triggered the phase transition. It is not difficult to understand that the hydrophilic additives introduced the opposite phase transition. With the goal of obtaining a stable preparation method of the hexagonal phase at room temperature, Amar-Yuli and Garti ${ }^{39}$ studied the effects of TAGs with different chain lengths (C2$\mathrm{C} 18$ ) on the effects of GMO/water system. It was found that TAG with very short chains like triacetin did not affect the phase behaviors, and the phases remained the $\mathrm{Q}_{2}$ phase and $\mathrm{L}_{2}$ phase. By contrast, TAG with middle chains like tricaprylin gave rise to the transformation of $\mathrm{L}_{2}$ phase to the $\mathrm{H}_{2}$ phase directly and it was the most stable and effective additive to trigger the transformation. This is because tricaprylin was incorporated between the GMO hydrophobic chains and filled the void volumes, and therefore increased the CPP value. Furthermore, in the other studies of Garti's group, tricaprylin was utilized to get the $\mathrm{H}_{2}$ phase. However, there was not much research on long-chain TAG since it would take at least one month to solvate the tails of GMO.

Hydrophobic additives such as vitamin E acetate (VitEA), ${ }^{78-83}$ tetradecane, ${ }^{81,84}$ hexadecane, ${ }^{22}$ triolein, ${ }^{81}$ limonene, ${ }^{21,65,81}$ cyclohexane ${ }^{65}$ and fatty acids ${ }^{25,85}$ have been reported and these additives are listed in Table 2 . VitEA is currently the most widely used additive for forming the hexagonal phase at room temperature. The work of Dong et al..$^{78}$ showed that the presence of hydrophobic VitEA in PT-water systems resulted in the formation of hexagonal phase at $25{ }^{\circ} \mathrm{C}$. The bulk LLC could be dispersed into nanoparticles while keeping the unique internal structures. These nanoparticles are called liposomes, hexosomes, and cubosomes, respectively. The dispersed hexosomes were successfully prepared by Kim $e t$ al. when adding
VitEA to PT-Pluronic F-127 (stabilizer)-water systems. ${ }^{79}$ The influence of hexadecane in PT and GMO was investigated respectively by Phan et al. ${ }^{22}$ SAXS data indicated that in GMOwater systems at $25^{\circ} \mathrm{C}, P n 3 m, \mathrm{H}_{2}$, micellar cubic phase $(F d 3 m)$, and inversed micelles $\left(\mathrm{L}_{2}\right)$ appeared when the concentration of hexadecane was $0-4 \%(\mathrm{w} / \mathrm{w}), 4-25 \%, 25-40 \%$ and $>40 \%$ respectively. However, with an increase in the concentration of hexadecane in PT-water systems, the phase transitioned from Pn3m to $\mathrm{H}_{2}$ to $\mathrm{L}_{2}$ without forming the micellar cubic phase.

The addition of hydrophilic additives to the system has two main purposes. One is introduction of a hydration-enhancing agent such as octyl glucoside (OG) ${ }^{86}$ sucrose stearate ${ }^{87}$ and diglycerol monooleate (DGMO) ${ }^{88}$ which can interact with the polar head of amphiphilics to increase the water channel dimensions and enable the LLC to encapsulate large molecules, such as proteins and peptides. Angelov et al. ${ }^{86}$ developed a novel cubic phase $(P n 3 m)$ with large water channels using OG as hydration-enhancing agent. SAXS showed that the phase structure did not change but the diameter of channel was twice of that of the normal phase. Negrini and Mezzenga ${ }^{87}$ enlarged the diameter of water channels in the monolinolein/water system by adding sucrose stearate. Studies showed that the phase transitioned from Pn $3 m$ to $\operatorname{Im} 3 m$ when the concentration of sucrose stearate was $10 \%(\mathrm{w} /$ w) and the size of water channels was increased from 3.85 to $12 \mathrm{~nm}$ with the concentration ranging from 0 to $25 \%(\mathrm{w} / \mathrm{w})$. That is because the sucrose stearate interacted with the polar head, reducing the CPP value and inducing the phase change from Pn $3 m$ to Im $3 m$.

Another purpose is the addition of solvents such as ethanol, ${ }^{61,89}$ propylene glycol, ${ }^{90}$ transcutol $^{91}$ etc. to form a lowviscosity lamellar phase and a micelle phase for injection administration. Chen et al. ${ }^{89}$ developed injectable in situ liquid crystals for intraarticular administration by adding ethanol to the PT-water system. The results indicated that isotropic solutions with low viscosity were formed.

Pharmaceutical compounds of different polarities have been encapsulated in lyotropic liquid crystals such as the hydrophilic drugs rhodamine B, ${ }^{92}$ acyclovir, ${ }^{93}$ lidocaine hydrochloride ${ }^{94}$ and the lipophilic drugs lidocaine ${ }^{94}$ cinnarizine, ${ }^{95}$ vitamin $\mathrm{K},{ }^{96}$ indomethacin, ${ }^{97}$ diclofenac sodium ${ }^{98,99}$ and so on. Similar to the additives, the entrapment of the hydrophilic drug causes 
Table 2 Effects of additives on the structure of LLC phases

\begin{tabular}{|c|c|c|c|}
\hline Composition & Addition & Type of phase & Reference \\
\hline GMO/water & Tricaprylin & $\mathrm{H}_{2}$ & 39 \\
\hline $\mathrm{PT} /$ water & VitEA & $\mathrm{H}_{2}$ & 78 \\
\hline Phosphatidylcholine/sorbitan monooleate/water & Tocopherol acetate & $\mathrm{L}_{\alpha} / \mathrm{H}_{2}$ & 80 \\
\hline $\mathrm{MLO} /$ water & Tetradecane & $\mathrm{H}_{2} / \mathrm{L}_{2} / F d 3 m$ & 84 \\
\hline GMO/water & Hexadecane & $\mathrm{H}_{2} / \mathrm{Q}_{2} / \mathrm{L}_{2} / F d 3 m$ & 22 \\
\hline $\mathrm{MLO} /$ water & Linoleic acid & $\operatorname{Im} 3 m / \mathrm{H}_{2}$ & 25 \\
\hline GMO/water & Oleic acid & $\mathrm{H}_{2} / \mathrm{L}_{2} / F d 3 m$ & 85 \\
\hline GMO/water & Octyl glucoside & Pn3m & 86 \\
\hline MLO/water & Sucrose stearate & $P n 3 m / \operatorname{Im} 3 m$ & 87 \\
\hline MLO/F-127/water & Diglycerol monooleate & Hexosomes/cubosomes $(\operatorname{Im} 3 m)$ & 88 \\
\hline $\mathrm{PT} /$ water & Ethanol & $\mathrm{L}_{2} / \mathrm{L}_{\alpha} / \mathrm{Q}_{2}$ & 61 \\
\hline
\end{tabular}

a phase change from the cubic phase to the lamellar phase, as opposed to the hydrophobic drugs.

\subsection{External factors}

3.2.1 Temperature. The phase behavior of LLC is sensitive to temperature. It has been found that, at room temperature, the lamellar phase and different types of reversed bicontinuous cubic phases are formed according to the GMO-water phase diagram. ${ }^{62}$ However, when the temperature rises to about $80{ }^{\circ} \mathrm{C}$, the cubic-to-reversed hexagonal phase transition occurs. PT gives rise to strikingly similar phase behavior in water to GMO where reversed hexagonal phase cannot form only if the temperature is raised to $50{ }^{\circ} \mathrm{C}$. As for MLO, the phase of lamellar crystal $\left(\mathrm{L}_{\mathrm{C}}\right), \mathrm{L}_{\alpha}, \operatorname{Ia} 3 d, \operatorname{Pn} 3 m$ are formed. Reverse hexagonal phase begins to appear when the temperature reaches $60{ }^{\circ} \mathrm{C} .{ }^{77}$ The thermal behavior of LLC can be well explained by CPP. The increasing temperature leads to a decrease of hydration of the lipid's polar head, and consequently a decrease in the crosssectional area. Increasing temperature also has a profound effect on hydrophobic tails, resulting in shorter tailed length but larger volume. In general, an increase in temperature leads to an increase in CPP.

The structure of lyotropic liquid crystals is extremely susceptible to temperature. In practical applications, sometimes, the prepared temperature of lyotropic liquid crystals is below $20{ }^{\circ} \mathrm{C}$. Qiu et al. ${ }^{62}$ prepared lyotropic liquid crystals at $0{ }^{\circ} \mathrm{C}$. The samples were then stored at $4{ }^{\circ} \mathrm{C}$ and $20{ }^{\circ} \mathrm{C}$ respectively. The results showed that the lamellar crystal was formed when stored at $4{ }^{\circ} \mathrm{C}$ and transformed into cubic phase at $20{ }^{\circ} \mathrm{C}$. The authors noted that the $\mathrm{L}_{\mathrm{C}}$ phase was formed in the lowtemperature region of the GMO/water phase diagram. However, it is metastable. Therefore, the prepared samples need to be stored at $20-25{ }^{\circ} \mathrm{C}$. For example, the samples can be placed in the incubator to maintain a constant temperature and avoid structural changes.

3.2.2 Pressure. Studies have shown that the effect of pressure on the structure of the liquid crystal phase is opposite to that of temperature, which means that the increase in pressure introduces the formation of the phases with negative spontaneous curvature $\left(H_{0}<0\right) .{ }^{100}$ Anan Yaghmur et al. ${ }^{101}$ utilized SAXS to investigate the impact of pressure and temperature on the behavior of inverted discontinuous $F d 3 m$ cubic phase formed by monoolein (GMO)/tetradecane (TC)/water systems. SAXS results revealed that the structure transitioned from the $F d 3 m$ to $F d 3 m$ coexisting with $\mathrm{H}_{\text {II }}$ and then to the $\mathrm{H}_{\text {II }}$ phase at $20{ }^{\circ} \mathrm{C}$ while pressure was varied from 1 to 1200 bar. Additionally, the lattice parameter of $F d 3 m$ rose from 23.04 to 24.17 nm with the pressure in the range of 1 to 900 bar while the lattice parameter of the $\mathrm{H}_{\mathrm{II}}$ phase increased from 6.20 to $6.51 \mathrm{~nm}$ with the pressure ranging from 900 to 1200 bar. Meanwhile, with the pressure rising from 1 to 750 bar at $7{ }^{\circ} \mathrm{C}$, the phase structure transitioned from $F d 3 m$ to the $\mathrm{H}_{\text {II }}$ phase, then to the $\mathrm{H}_{\text {II }}$ phase coexisting with $P n 3 m$ and finally to $P n 3 m$, respectively.

3.2.3 Light. Light can be used to control the phase transition of LLC. There are two main ways to achieve this goal. One of these is incorporating gold nanorods in LLCs. ${ }^{27-30}$ Gold nanoparticles can absorb the light energy and convert it into heat, which promotes the structural transformation. Fong et al. ${ }^{30}$ embedded gold nanorods in a LLC prepared by PT and achieved the phase transformation from the cubic phase to the hexagonal phase by irradiation of NIR laser light, which in turn control the release rate of model drug ${ }^{14} \mathrm{C}$ glucose. Encapsulating photochromic molecules such as spiropyran (SP), spirooxazine (SOX) and spiropyran laurate (SPL) is another way to introduce phase transformation. ${ }^{31,32}$ These photochromic molecules can bind strongly to lipid molecules. Upon exposure to UV irradiation, the lattice parameters of the systems undergo changes, indicating that the photochromic molecules can disrupt lipid packing.

3.2.4 Magnetic fields. Incorporating magnetic nanoparticles, such as $\mathrm{Fe}_{3} \mathrm{O}_{4}$ nanoparticles, offers an excellent way to control the transport properties of mesophases. ${ }^{33,34}$ Specifically, under the external magnetic field, $\mathrm{Fe}_{3} \mathrm{O}_{4}$ nanoparticles will be arranged in a straight line which enhances the solute mobility 
in the aligned direction. The $\mathrm{Fe}_{3} \mathrm{O}_{4}$ nanoparticles are hashed or arranged vertically without magnetic field. Mezzenga et al. ${ }^{34}$ developed a reverse hexagonal phase doped with magnetic iron oxide nanoparticles and studied the diffusion of two hydrophilic drugs glucose and caffeine. In the case of glucose, the hexagonal phase parallel to the diffusion chamber showed more than 8-fold increase in the diffusion coefficient compared with that of the hexagonal phase aligned in the vertical direction. This experiment also demonstrated that not only the size of water domains and the symmetry but also the direction of the domains plays a role in drug diffusion.

\section{The correlation between structure and drug diffusion}

LLC is capable of solubilizing drugs with different polarities (hydrophilic, hydrophobic, amphiphilic) due to the unique internal structures. The position of these drugs are different. Generally, hydrophilic drugs are located close to the polar head or in the water channels, while hydrophobic drugs are loaded in the lipid layer, and amphiphilic drugs at the interface. Furthermore, according to previous research, the release of drugs from LLC conformed to Higuchi diffusion kinetics, where the cumulative release of drugs was proportional to the square root of the time. ${ }^{\mathbf{1 0 2 , 1 0 3}}$ The Higuchi diffusion equation is given as follow:

$$
\frac{M_{t}}{M_{\infty}}=K_{\mathrm{H}} \sqrt{t}
$$

where $M_{t}$ and $M_{\infty}$ are the amount of drug released at time $t$ and at infinite time respectively, and $K_{\mathrm{H}}$ is the release rate constant. ${ }^{104}$ However, the release rate of the solubilized drugs were related to many factors, such as the polarities of drugs, ${ }^{65,87,105}$ the molecular weight of drugs, ${ }^{87,106}$ and the internal structures, ${ }^{22}$ etc. In fact, the drug diffusion kinetics depend in a complex way on the geometry, as demonstrated theoretically and experimentally by Antognini et $a{ }^{107}$ In the case of the lamellar phase, the flat water layer is separated by hydrophobic bilayers. As for the hexagonal phase, there exist onedimensional parallel water cylinders, and the water channels of the cubic phase are continuous but nonintersecting. Moreover, hydrophilic drugs are located in the polar core of the inverse micellar cube. Consequently, the release rates of loaded drugs are in the order as follows: $D_{\text {lamellar phase }}>D_{\text {cubic phase }}>$ $D_{\text {hexagonal phase }}>D_{\text {micellar cubic }} \cdot{ }^{108}$ Many researchers have studied the release of drugs with different properties from different liquid crystal structures and we will discuss this in the following sections.

\subsection{Release of hydrophilic drugs}

Phan et $a .^{22}$ investigated the release rate of model hydrophilic drug glucose in four GMO-based mesophases which were the reversed bicontinuous cubic phase $\left(\mathrm{V}_{2}\right)$, the inverse hexagonal phase $\left(\mathrm{H}_{2}\right)$, the micellar cubic phase $\left(\mathrm{I}_{2}\right)$, and the inverse miceller phase $\left(\mathrm{L}_{2}\right)$ respectively. The results indicated that the drug release in four mesophases followed first-order diffusion kinetics. The study demonstrated that LLC structures could be used to control the rate of release. The release rates of glucose were in the order as follow: $\mathrm{V}_{2}>\mathrm{L}_{2}>\mathrm{H}_{2}>\mathrm{I}_{2}$. Lee et al. ${ }^{108}$ explored the relationship between hydrophilic drugs of different molecular weight $\left({ }^{14} \mathrm{C}\right.$-glucose, Allura Red and FITC-dextrans) and the cubic phase formed by GMO ( $\left.\mathrm{Q}_{\mathrm{GMO}}\right)$, the cubic phase formed by $\mathrm{PT}\left(\mathrm{Q}_{\mathrm{PT}}\right)$ and the hexagonal phase formed by PT and VE $\left(\mathrm{H}_{\mathrm{PT}}\right)$. It was found that the release rate of drugs was the greatest in $\mathrm{Q}_{\mathrm{GMO}}$, followed by $\mathrm{Q}_{\mathrm{PT}}$ and $\mathrm{H}_{\mathrm{PT}}$. In addition, molecular weight also affected the release of drugs and the release of those with a small molecular weight $\left({ }^{14} \mathrm{C}\right.$-glucose $)$ was the fastest. Furthermore, there have been many studies on the in vitro release of macromolecules such as protein drugs and release processes that follow Higuchi diffusion kinetics.

\subsection{Release of hydrophobic drugs}

Hydrophobic drugs irinotecan, ${ }^{109}$ paclitaxel, ${ }^{87}$ caffeine,${ }^{65}$ proflavine, ${ }^{65}$ diazepam, ${ }^{\mathbf{1 1 0}}$ propofol, ${ }^{111}$ rifampicin ${ }^{\mathbf{1 1 2}}$ were used to study the release behavior. In vitro release experiments showed that the release of encapsulated hydrophobic drugs followed Higuchi diffusion kinetics too. Boyd et al. ${ }^{109}$ focused their research on the release of irinotecan in hexosome dispersions. The results showed that the release of irinotecan was controlled by diffusion, despite being essentially instantaneous.

\section{Conclusion and outlook}

LLC systems have received considerable attention and research as drug carriers. In recent years, more and more researchers are focusing on the structure of LLCs and the release mechanism of drugs, especially R. Mezzenga's research team.

This article mainly reviews the influencing factors on LLC structures and the relationship between drugs diffusion and structures. Fully understanding the factors that affect the structure of lyotropic liquid crystals is conducive to controlling the preparation conditions and obtaining a stable liquid crystal phase. Furthermore, we can utilize these factors to design intelligent LLC carriers. Additionally, studying the relationship between drug release and structures can provide a reference for encapsulating different types of drugs.

Certainly, there are many difficulties to overcome for the clinical application of lyotropic liquid crystals, among which are the biocompatibility and safety of lipid materials. It is reported that GMO cause hemolysis, especially for intravenous (IV) administration. ${ }^{113}$ PEGylation (polyethylene glycol, PEG) can be used to improve the hemocompatibility of MO-based systems. ${ }^{114,115}$ Furthermore, some new materials such as dioleoyl phosphatidylethanolamine (DOPE) ${ }^{\mathbf{1 1 6}}$ glycerol dioleate (GDO) and soy phosphatidylcholine (SPC) ${ }^{\mathbf{1 1 7}}$ can reduce hemolysis. Nevertheless, LLC is still considered a good candidate for drug delivery, and further research is expected for deeper exploration of applications of LLC as drug carriers.

\section{Conflicts of interest}

The authors declare that there is no conflict of interests regarding the publication of this paper. 


\section{Acknowledgements}

This work was supported by the National Natural Science Foundation of China (81274099).

\section{References}

1 S. B. Rizwan, B. J. Boyd, T. Rades and S. Hook, Expert Opin. Drug Delivery, 2010, 7(10), 1133.

2 J. Clogston and M. Caffrey, J. Controlled Release, 2005, $107(1), 97$.

3 T. Kaasgaard and C. J. Drummond, Phys. Chem. Chem. Phys., 2006, 8(43), 4957-4975.

4 J. C. Shah, Y. Sadhale and D. M. Chilukuri, Adv. Drug Delivery Rev., 2001, 47(2), 229-250.

5 A. Angelova, B. Angelov, R. Mutafchieva, S. Lesieur and P. Couvreur, Acc. Chem. Res., 2011, 44(2), 147-156.

6 L. Sagalowicz, R. Mezzenga and M. E. Leser, Curr. Opin. Colloid Interface Sci., 2006, 11(4), 224-229.

7 D. Libster, A. Aserin, E. Wachtel, G. Shoham and N. Garti, J. Colloid Interface Sci., 2007, 308(2), 514.

8 V. Luzzati, Curr. Opin. Struct. Biol., 1997, 7(5), 661-668.

9 C. J. Drummond and C. Fong, Curr. Opin. Colloid Interface Sci., 1999, 4(6), 449-456.

10 L. Sagalowicz, M. E. Leser, H. J. Watzke and M. Michel, Trends Food Sci. Technol., 2006, 17(5), 204-214.

11 S. Z. Mohammady, M. Pouzot and R. Mezzenga, Biophys. J., 2009, 96(4), 1537-1546.

12 P. S. Pershan, Phys. Today, 1982, 35(5), 34-39.

13 J. Barauskas, A. M. Johnsson and F. Tiberg, Nano Lett., 2005, 5(8), 1615-1619.

14 T. H. Nguyen, T. Hanley, C. J. Porter and B. J. Boyd, J. Controlled Release, 2011, 153(2), 180.

15 K. Holmberg, Pigm. Resin Technol., 2002, 10(5), 383-402.

16 K. Larsson, J. Phys. Chem., 1989, 93(21), 512-513.

17 K. Larsson, Curr. Opin. Colloid Interface Sci., 2000, 5(1), 6469.

18 R. Laughlin, J. Am. Chem. Soc., 1995, 117(42), 10603.

19 W. K. Fong, T. Hanley and B. J. Boyd, J. Controlled Release, 2009, 135(3), 218

20 H. Chung, J. Kim, J. Y. Um, I. C. Kwon and S. Y. Jeong, Diabetologia, 2002, 45(3), 448-451.

21 M. Pouzot, R. Mezzenga, M. Leser, L. Sagalowicz, S. Guillot and O. Glatter, Langmuir, 2007, 23(19), 9618-9628.

22 S. Phan, W. K. Fong, N. Kirby, T. Hanley and B. J. Boyd, Int. J. Pharm., 2011, 421(1), 176-182.

23 C. M. Chang and R. Bodmeier, J. Controlled Release, 1997, 46(3), 215-222.

24 T. K. Kwon and J. C. Kim, Drug Dev. Ind. Pharm., 2011, 37(1), 56.

25 R. Negrini and R. Mezzenga, Langmuir, 2011, $27(9), 5296$.

26 A. Zabara, R. Negrini, O. Onaca-Fischer and R. Mezzenga, Small, 2013, 9(21), 3602-3609.

27 W. K. Fong, T. L. Hanley, B. Thierry, N. Kirby and B. J. Boyd, Langmuir, 2010, 26(26), 6136-6139.
28 W. K. Fong, T. L. Hanley, B. Thierry, N. Kirby, L. J. Waddington and B. J. Boyd, Langmuir, 2012, 28(40), 14450.

29 A. Yaghmur, L. Paasonen, M. Yliperttula, A. Urtti and M. Rappolt, J. Phys. Chem. Lett., 2010, 1(6), 962-966.

30 W. K. Fong, T. L. Hanley, B. Thierry, A. Hawley, B. J. Boyd and C. B. Landersdorfer, J. Controlled Release, 2016, 228, 67.

31 K. J. Tangso, W. K. Fong, T. Darwish, N. Kirby, B. J. Boyd and T. L. Hanley, J. Phys. Chem. B, 2013, 117(35), 1020310210.

32 W. K. Fong, N. Malic, R. A. Evans, A. Hawley, B. J. Boyd and T. L. Hanley, Biointerphases, 2012, 7(1-4), 1-5.

33 J. J. Vallooran, S. Bolisetty and R. Mezzenga, Adv. Mater., 2011, 23(34), 3932.

34 J. J. Vallooran, R. Negrini and R. Mezzenga, Langmuir, 2013, 29(4), 999-1004.

35 B. J. Boyd, D. V. Whittaker, S. M. Khoo and G. Davey, Int. J. Pharm., 2006, 309(1-2), 218.

36 P. Wadsten-Hindrichsen, J. Bender, J. Unga and S. Engström, J. Colloid Interface Sci., 2007, 315(2), 701-713.

37 J. Barauskas and T. Landh, Langmuir, 2003, 19(23), 95629565.

38 M. Shah and A. Paradkar, Eur. J. Pharm. Biopharm., 2007, 67(1), 166-174.

39 I. Amar-Yuli and N. Garti, Colloids Surf., B, 2005, 43(2), 72. 40 A. Asokan and M. J. Cho, J. Pharm. Sci., 2002, 91(4), 903-913.

41 L. E. Gerweck and K. Seetharaman, Cancer Res., 1996, 56(6), 1194-1198.

42 C. Speziale, M. L. Salvati, C. Manatschal, E. M. Landau and R. Mezzenga, Proc. Natl. Acad. Sci. U. S. A., 2016, 113(27), 7491.

43 C. Speziale, A. F. Zabara, C. J. Drummond and R. Mezzenga, ACS Nano, 2017, 11(11), 11687-11693.

44 S. T. Hyde, Curr. Opin. Solid State Mater. Sci., 1996, 1, 653.

45 P. J. Collings, M. Hird and C. C. Huang, Am. J. Phys., 1997, 66(6), 551.

46 N. Garti, D. Libster and A. Aserin, Food Funct., 2012, 3(7), 700.

47 K. Larsson, Curr. Opin. Colloid Interface Sci., 2009, 14(1), 1620.

48 V. Luzzati and F. Husson, J. Cell Biol., 1962, 12(2), 207.

49 B. D. Kruijff, Nature, 1997, 386, 129-130.

50 D. C. Turner, Z. G. Wang, S. M. Gruner, D. A. Mannock and R. N. Mcelhaney, J. Phys. B: At., Mol. Opt. Phys., 1992, 2(11), 2039-2063.

51 J. Briggs, H. Chung and M. Caffrey, J. Phys. B: At., Mol. Opt. Phys., 1996, 6(5), 723-751.

52 J. M. Seddon, Biochim. Biophys. Acta, 1990, 1031(1), 1.

53 U. S. Schwarz and G. Gompper, Langmuir, 2001, 17(7), 20842096.

54 R. Mezzenga, C. Meyer, C. Servais, A. I. Romoscanu, L. Sagalowicz and R. C. Hayward, Langmuir, 2005, 21(8), 3322-3333.

55 J. M. Seddon, Biochemistry, 1990, 29(34), 7997-8002.

56 J. M. Seddon, J. Robins, T. G. Krzywicki and H. Delacroix, Phys. Chem. Chem. Phys., 2000, 2(2), 4485-4493. 
57 I. Martiel, L. Sagalowicz and R. Mezzenga, Langmuir, 2013, 29(51), 15805-15812.

58 W. B. Lee, R. Mezzenga and G. H. Fredrickson, Phys. Rev. Lett., 2007, 99(18), 187801.

59 J. Israelachvili, Colloids Surf., A, 1994, 91, 1-8.

60 C. Fong, T. Le and C. J. Drummond, Chem. Soc. Rev., 2012, 41(3), 1297-1322.

61 K. Han, X. Pan, M. W. Chen, R. Wang, Y. H. Xu, M. Feng, et al., Eur. J. Pharm. Sci., 2010, 41(5), 692.

62 H. Qiu and M. Caffrey, Biomaterials, 2000, 21(3), 223-234.

63 J. C. Li, N. Zhu, J. X. Zhu, W. J. Zhang, H. M. Zhang, Q. Q. Wang, et al., Med. Sci. Monit., 2015, 21, 3298-3310.

64 I. Martiel, L. Sagalowicz, S. Handschin and R. Mezzenga, Langmuir, 2014, 30(48), 14452-14459.

65 I. Martiel, N. Baumann, J. J. Vallooran, J. Bergfreund, L. Sagalowicz and R. Mezzenga, J. Controlled Release, 2015, 204, 78.

66 B. J. Boyd, S. M. Khoo, D. V. Whittaker, G. Davey and C. J. Porter, Int. J. Pharm., 2007, 340(1-2), 52.

67 B. M. Bertelsen, K. S. Korsholm, F. Rose, P. Nordly, H. Franzyk, P. Andersen, et al., RSC Adv., 2013, 3(43), 20673-20683.

68 M. Sjölund, G. Lindblom, L. Rilfors and G. Arvidson, Biophys. J., 1987, 52(2), 145-153.

69 P. O. Eriksson and G. Lindblom, Biophys. J., 1993, 64(1), 129-136.

70 L. T. Boni and S. W. Hui, Biochim. Biophys. Acta, 1983, 731(2), 177-185.

71 B. Tenchov, M. Rappolt, R. Koynova and G. Rapp, Biochim. Biophys. Acta, 1996, 1285(1), 109-122.

72 B. Tenchov, R. Koynova and G. Rapp, Biophys. J., 1998, 75(2), 853-866.

73 M. H. And and H. Minamikawa, Langmuir, 1996, 12(6), 1658-1665.

74 C. Fong, D. Wells, I. Krodkiewska, J. Booth and P. G. Hartley, J. Phys. Chem. B, 2007, 111(6), 1384.

75 K. Didehban and H. Namazib, Eur. Polym. J., 2010, 46(9), 1923-1931.

76 S. Milak and A. Zimmer, Int. J. Pharm., 2015, 478(2), 569587.

77 R. Mezzenga, P. Schurtenberger, A. Burbidge and M. Michel, Nat. Mater., 2005, 4(10), 729-740.

78 Y. D. Dong, I. Larson, T. Hanley and B. J. Boyd, Langmuir, 2006, 22(23), 9512-9518.

79 D. H. Kim, S. Lim, J. Shim, J. E. Song, J. S. Chang, K. S. Jin, et al., ACS Appl. Mater. Interfaces, 2015, 7(36), 20438-20446.

80 Y. M. Báez-Santos, A. Otte, E. A. Mun, B. K. Soh, C. G. Song, Y. N. Lee, et al., Int. J. Pharm., 2016, 514(1), 314-321.

81 L. Sagalowicz, S. Guillot, S. Acquistapace, B. Schmitt, M. Maurer, A. Yaghmur, et al., Langmuir, 2013, 29(26), 8222-8232.

82 L. Bitancherbakovsky, I. Yuliamar, A. Aserin and N. Garti, Langmuir, 2010, 26(5), 3648-3653.

83 L. Bitancherbakovsky, I. Yuliamar, A. Aserin and N. Garti, Langmuir, 2009, 25(22), 13106-13113.

84 A. Yaghmur, C. L. De, S. Salentinig, L. Sagalowicz, M. E. Leser and O. Glatter, Langmuir, 2006, 22(2), 517-521.
85 J. Borné, A. T. Nylander and A. Khan, Langmuir, 2001, 17(25), 7742-7751.

86 B. Angelov, A. Angelova, V. M. Garamus, G. Lebas, S. Lesieur, M. Ollivon, et al., J. Am. Chem. Soc., 2007, 129(44), 13474.

87 R. Negrini and R. Mezzenga, Langmuir, 2012, 28(47), 1645516462.

88 A. Yaghmur, L. D. Campo, L. Sagalowicz, M. E. Leser and O. Glatter, Langmuir, 2006, 22(24), 9919-9927.

89 Y. Chen, X. Liang, P. Ma, Y. Tao, X. Wu, X. Chu, et al., AAPS PharmSciTech, 2015, 16(4), 846-854.

90 X. Jiang, X. Liang, S. Wang, J. Guo, Y. Tao and S. Gui, J. Pharm. Sci., 2017, 72(5), 249-256.

91 I. A. Yuli, E. Wachtel, D. E. Shalev, A. Aserin and N. Garti, J. Phys. Chem. B, 2008, 112(13), 3971-3982.

92 J. Bender, C. Simonsson, M. Smedh, S. Engström and M. B. Ericson, J. Controlled Release, 2008, 129(3), 163-169.

93 L. S. Helledi and L. Schubert, Drug Dev. Ind. Pharm., 2001, 27(10), 1073.

94 S. Engström and L. Engström, Int. J. Pharm., 1992, 79(2-3), 113-122.

95 B. J. Boyd, S. M. Khoo, D. V. Whittaker, G. Davey and C. J. Porter, Int. J. Pharm., 2007, 340(1-2), 52-60.

96 L. B. Lopes, F. F. Speretta and M. V. Bentley, Eur. J. Pharm. Sci., 2007, 32(3), 209.

97 E. Esposito, R. Cortesi, M. Drechsler, L. Paccamiccio, P. Mariani, C. Contado, E. Stellin, E. Menegatti, F. Bonina and C. Puglia, Pharm. Res., 2005, 22(12), 2163-2173.

98 D. Yariv, R. Efrat, D. Libster, A. Aserin and N. Garti, Colloids Surf., B, 2010, 78(2), 185-192.

99 M. Cohenavrahami, A. Aserin and N. Garti, Colloids Surf., B, 2010, 77(2), 131-138.

100 J. M. Seddon, A. M. Squires, C. E. Conn, O. Ces, A. J. Heron, X. Mulet, et al., Philos. Trans. R. Soc., A, 2006, 364(1847), 2635-2655.

101 A. Yaghmur, M. Kriechbaum, H. Amenitsch, M. Steinhart, P. Laggner and M. Rappolt, Langmuir, 2010, 26(2), 11771185.

102 S. Dash, P. N. Murthy, L. Nath and P. Chowdhury, Acta Pol. Pharm., 2010, 67(3), 217.

103 P. Costa and J. M. S. Lobo, Eur. J. Pharm. Sci., 2001, 13(2), 123.

104 P. Costa, D. C. Ferreira and J. M. S. Lobo, Rev. Port. Farm., 1996, 46(1), 2-8.

105 E. Rosenbaum, S. Tavelin and L. B. Johansson, Int. J. Pharm., 2010, 388(1), 52-57.

106 A. R. Ahmed, A. Dashevsky and R. Bodmeier, Eur. J. Pharm. Biopharm., 2008, 70(3), 765-769.

107 L. M. Antognini, S. Assenza, C. Speziale and R. Mezzengaa, J. Chem. Phys., 2016, 145(8), 084903.

108 K. W. Lee, T. H. Nguyen, T. Hanley and B. J. Boyd, Int. J. Pharm., 2009, 365(1-2), 190-199.

109 B. J. Boyd, D. V. Whittaker, S. M. Khoo and G. Davey, Int. J. Pharm., 2006, 318(1-2), 154-162.

110 T. H. Nguyen, T. Hanley, C. J. Porter, I. Larson and B. J. Boyd, J. Pharm. Pharmacol., 2010, 62(7), 844-855. 
111 M. Johnsson, J. Barauskas, A. Norlin and F. Tiberg, J. Nanosci. Nanotechnol., 2006, 6(9-10), 3017-3024.

112 R. Parmar, R. Misra and S. Mohanty, Colloids Surf., B, 2015, 129, 198-205.

113 J. C. Bode, J. Kuntsche, S. S. Funari and H. Bunjes, Int. J. Pharm., 2013, 448(1), 87-95.

114 M. S. Freag, Y. S. R. Elnaggar, D. A. Abdelmonsif and O. Y. Abdallah, Int. J. Nanomed., 2016, 11, 4799-4818.
115 A. Angelova, B. Angelov, M. Drechsler, V. M. Garamus and S. Lesieur, Int. J. Pharm., 2013, 454(2), 625-632.

116 J. Barauskas, M. Johnsson and F. Tiberg, Nano Lett., 2005, 5(8), 1615-1619.

117 J. Barauskas, C. Cervin, M. Jankunec, M. Špandyreva, K. Ribokaite, F. Tiberg and M. Johnsson, Int. J. Pharm., 2010, 391(1-2), 284-291. 